EPJ Web of Conferences 92,02074 (2015)

DOI: $10.1051 /$ epjconf/ 20159202074

(C) Owned by the authors, published by EDP Sciences, 2015

\title{
The impact of plasma induced flow on the boundary layer in a narrow channel
}

\author{
P. Procházka ${ }^{1, \mathrm{a}}$ and V. Uruba ${ }^{1}$ \\ ${ }^{1}$ Academy of Sciences of the Czech Republic, Institute of Thermomechanics, Dolejškova 1402/5, 18200 Prague, Czech \\ Republic
}

\begin{abstract}
The induced flow generated by dielectric barrier discharge (DBD) actuator working in steady and unsteady regime will be used to modify properties of naturally developed boundary layer (BL) in short and long rectangular perspex channel which is connected to the blow-down wind tunnel. The actuator is placed in spanwise configuration and the inlet velocities will range between 5 and $20 \mathrm{~m} \cdot \mathrm{s}^{-1}$. Previously, mean flow field and statistical quantities were subjugated to investigation. In this paper, there will be presented dynamical features of the BL. Oscillation pattern decomposition (OPD) of influenced flow field and frequency analysis will be presented. These results should be taken into account regarding to use in the flow around a bluff body.
\end{abstract}

\section{Introduction}

Plasma actuators still remain in the center of interest for many researcher groups and they are still improving. The shape of electrodes is improving (e.g. [1]) or there is a development of actuators working principle itself. However up to the present time, there are just several studies dealing with unsteady regime of plasma actuators which could be more convenient for purpose of flow control than steady regime when the ionic wind is produced continuously. This article reveals the interaction of ionic wind generated by plasma dielectric barrier discharge (DBD) actuator with boundary layer (BL). Interaction of coherent structures generated during unstable regime is investigated by OPD decomposition which can decompose both toposes and chronoses and even related frequencies present in the flow field.

The flow field which is generated by DBD actuator in steady air was studied in detail and presented in [2]. Steady regime is characterized by wall-jet-like flow (with maximal mean velocities around $2 \mathrm{~m} \cdot \mathrm{s}^{-1}$ ) and its nature is given, of course, by actuator geometry and by the electrical parameters - voltage and frequency of alternating current (AC). The unsteady regime occurs after that the modulation is applied - then another two electrical parameters (modulation frequency and duty cycle) can be incorporated which strongly affects the nature of ionic wind. There are present vortical structures so called vortex train due to discontinuous ionic wind. The dependency of properties of vortices on modulation frequency and duty cycle was investigated and a physical model describing this phenomenon was presented which is to be used for control over GG (GlaubertGoldschmiedt) body. That model delineates the main parameters of vortex motion as convective velocity as well as changing geometry of vortices as vortex radius or circulation.

Before an experiment of plasma actuation over GG body will be held, there was decided to check how this actuator can affect much simpler case. This article will report about the influence of plasma ionic wind on developed BL from both statistical and dynamical point of view. Several velocity profiles in different position and obtained under various condition will be plotted as well as comparison of velocity variance. The dynamics of flow control will be observed via OPD when new frequencies present in the flow under control will be searched. Just experimental approach was proposed to solve this problem due to complex character of this task.

\section{Experimental setup}

\subsection{Actuator description}

Wire type of plasma DBD actuator [3] is adjusted version of simple DBD and consists of two electrodes separated by a dielectric layer. Powered and air-exposed electrode is actually very thin wire $(30 \mu \mathrm{m})$ which is normally used for hot-wire anemometry. The lower electrode is grounded and it is very thin layer of gold glaze. The silica glass plate was used as a dielectric layer because is able

Corresponding author: prochap@it.cas.cz 
to withstand very high voltage value with relatively thin layer. The horizontal gap between electrodes is set to two millimetres.

The actuator had to be reconstructed in order to minimize the disruption of boundary layer. The holder of wire itself and of spiral spring was rebuilt to not affect the free flow. There are just two small anchor points for wire mechanism now, so actuator can be used in spanwise configuration for both orientation of plasma wind (ensuring by turning actuator around). Since the wire electrode exhibits right in the middle of actuator plate $(150 \times 100 \mathrm{~mm})$, the coordinate system remains the same for both directions.

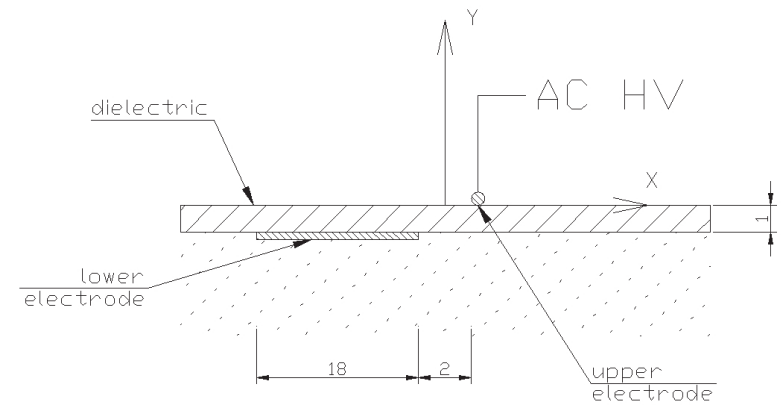

Figure 1. Plasma DBD actuator - wire type

\subsection{Power source}

The power source which was introduced in study [4] is used for generation of high-frequency high-voltage waveform which can be modulated if it is desirable. The nominal value of voltage is $12 \mathrm{kV}$ and the frequency is set to $16 \mathrm{kHz}$. If the plasma actuator is supplied by this signal, wall-jet-like flow is produced. The rectangular modulation is performed by shut-down function of power source. Then there are another two electrical parameters modulation frequency and duty cycle - which resulting in the series of vortices that are present in the generated flow. The modulation frequency ranges from units of Hertz to tens of Hertz. Previously, it was observed that the vortex train starts to be similar to the continuous wall jet for frequencies higher than $80 \mathrm{~Hz}$. Duty cycle inversely expresses what rate of one period the plasma discharge is on. Also duty cycle of $30 \%$ means that plasma discharge is on for $70 \%$ of one period which is given by modulation frequency.

Table 1. Reynolds number for long channel.

\begin{tabular}{|c|c|c|}
\hline & $\mathbf{R e}_{\mathbf{x}}$ & $\mathbf{R e}_{\boldsymbol{\delta}}$ \\
\hline $5 \mathrm{~m} \cdot \mathrm{s}^{-1}$ & $750 \cdot 10^{3}$ & $18 \cdot 10^{3}$ \\
\hline $10 \mathrm{~m} \cdot \mathrm{s}^{-1}$ & $1500 \cdot 10^{3}$ & $34 \cdot 10^{3}$ \\
\hline $20 \mathrm{~m} \cdot \mathrm{s}^{-1}$ & $3000 \cdot 10^{3}$ & $55 \cdot 10^{3}$ \\
\hline
\end{tabular}

\subsection{Experimental layout}

The impacted boundary layer was developed inside a perspex channel with cross-section of $100 \times 250 \mathrm{~mm}$. The channel was connected to the blow-down wind tunnel [5] and its length was $3000 \mathrm{~mm}$ as the main case. The actuator was mounted inside it in spanwise configuration so that the distance from the inlet edge of the channel to the wire electrode was $2275 \mathrm{~mm}$. The actuator can be mounted in opposite way to generate ionic wind opposite to the free flow inside the channel (see figure 2).

The wall of the channel was smooth without any turbulizators so the parameters of the BL were changed just by velocity of the wind tunnel. The velocities were set to 5,10 and $20 \mathrm{~m} \cdot \mathrm{s}^{-1}$ which correspond to conventional boundary layer thickness of 54, 51 and 41 $\mathrm{mm}$, respectively. These thicknesses were determined from PIV data in the position of wire electrode for the base case (plasma is off). There are Reynolds number based on thickness and horizontal distance in the table 1 . A second channel with different length was used as a comparative case where the length of channel was 1000 $\mathrm{mm}$ and wire electrode was placed $280 \mathrm{~mm}$ downstream the channel inlet. Since the thickness of such boundary layer remains rather small (approx. four millimetres), the PIV is not very convenient for thickness determination in scales for which this experiment was conducted.

The experiment itself was carried out under several various configuration of plasma actuator. The base case when the plasma is off all the time was considered as a reference case. The other regime is continuous wall jet which is produced without modulation of voltage signal. The last one is unsteady regime when vortex train is produced via modulation parameters. Tested frequencies were 10,20 and $50 \mathrm{~Hz}$, all for duty cycle of $30 \%$.

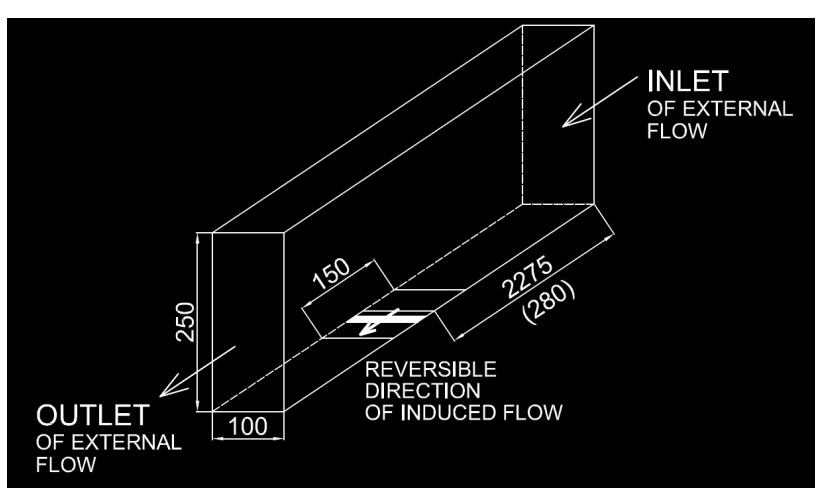

Figure 2. Experimental layout, long and short channel

\subsection{Measurement technique}

The time-resolved Particle Image Velocimetry (PIV) was used as main measurement technique. Laser New Wave Pegasus Nd:YLF is a double-head pulse laser with a cylindrical optics and its wavelength is directly $257 \mathrm{~nm}$. Maximal frequency of laser is $10 \mathrm{kHz}$ and shot energy in one pulse is $10 \mathrm{~mJ}$ (for $1 \mathrm{kHz}$ frequency). The CCD camera is Phantom V711 type and has maximal resolution of $1280 \times 800$ pixels which can be reduced 
resulting in higher sampling frequency. Standard maximal frequency is $6 \mathrm{kHz}$ (3000 double-images per second) and total memory of camera is $8 \mathrm{~GB}$ of acquired data.

The acquisition frequency differed depending on whether the statistical or dynamical phenomenon was measured. For data acquisition of statistics, the sampling frequency was set to $100 \mathrm{~Hz}$ with time of measurement at least ten seconds. On the other hand, to fulfill the Nyquist criterion, frequency was set to $2 \mathrm{kHz}$ to not miss all fastest processes during dynamical part of measurement.

\section{Results}

\subsection{Statistical approach}

Since the horizontal resolution of CCD camera is not sufficient, the observed area of BL was divided into five sections. Then the camera images were acquired in position $0,1,2,3,4$ and 5 resulting in downstream distance from wire electrode $0,60,150,250$ and $350 \mathrm{~mm}$, respectively. The position 0 was set to capture the flow instabilities in the vicinity and upstream the wire electrode.

The effect of plasma actuation can be seen very well from velocity profiles gained from mean flow fields. The profiles are normalized according to the external flow to be able to compare different cases. The figure 3 shows the profile of naturally developed BL (base case - red color) with steady regime of actuation (plasma is on) and unsteady case (plasma is generated by $10 \mathrm{~Hz}$ ). It can be seen very clearly that velocities close to the surface are accelerated about $8 \%$ for the case of the lowest velocity of external flow. The impact of plasma became weaker for velocity of $10 \mathrm{~m} \cdot \mathrm{s}^{-1}$ and the advance is almost negligible for velocity of $20 \mathrm{~m} \cdot \mathrm{s}^{-1}$ where this actuator cannot produce sufficient net body force to significantly accelerate the flow inside the BL. The comparison of three modulation frequencies is not plotted below, but the most promising results occurs for the highest modulation frequency when the vortex train starts to be similar to the continuous wall-jet-like flow which is more effective.

From statistical point of view, it is very effective to compare the standard deviations of velocity also. It seems that plasma actuation can stabilize the flow very close to the surface by adding necessary momentum. However, this effect is obvious for all points of BL. This stabilization of flow is to be promising for the case of delaying of separation point.

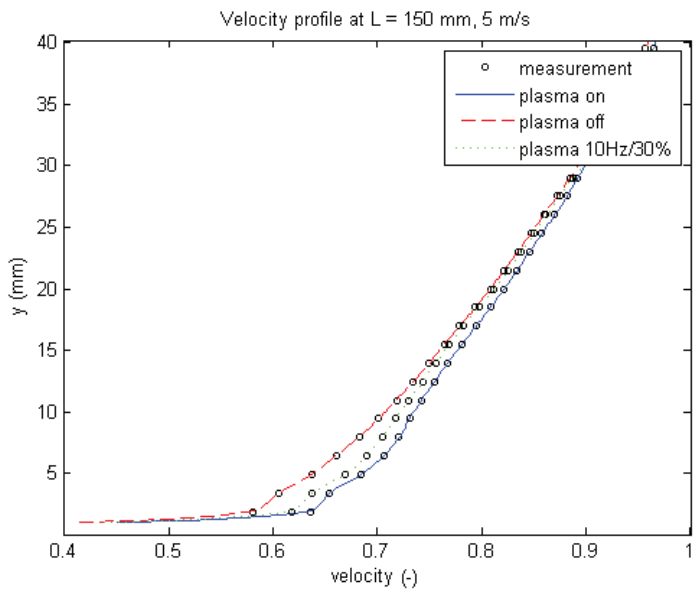

Figure 3. Velocity profiles improvement in second position, plasma actuation in corresponding direction, long channel

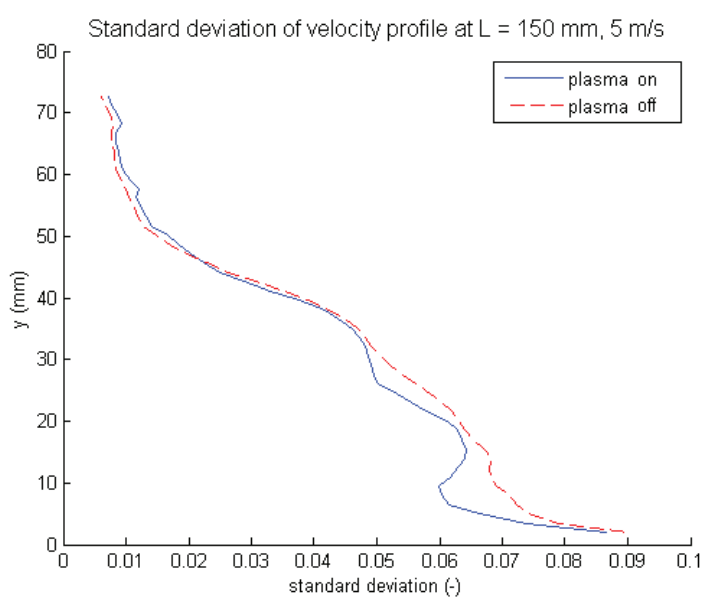

Figure 4. Velocity standard deviation, plasma actuation in corresponding direction, long channel

The great impact of plasma induced ionic wind is well useful, if we want to decelerate the velocities and thus to evoke early separation of the flow. Of course, the plasma actuator is then oriented in opposite direction. There is plotted the profile in the same position from wire electrode downstream $(\mathrm{L}=150 \mathrm{~mm})$ for the very same velocity in the figure 5 . The effect of deceleration is in the same order as the effect of acceleration.

Also, these phenomena were observed for the not well-developed BL in short channel. Massive deceleration of the flow about approximately $20 \%$ was found out for all positions of camera (figure 6 in $\mathrm{L}=150$ $\mathrm{mm}$ ). The unsteady regime (green color) is more effective which does not correspond with the results from longer channel. 


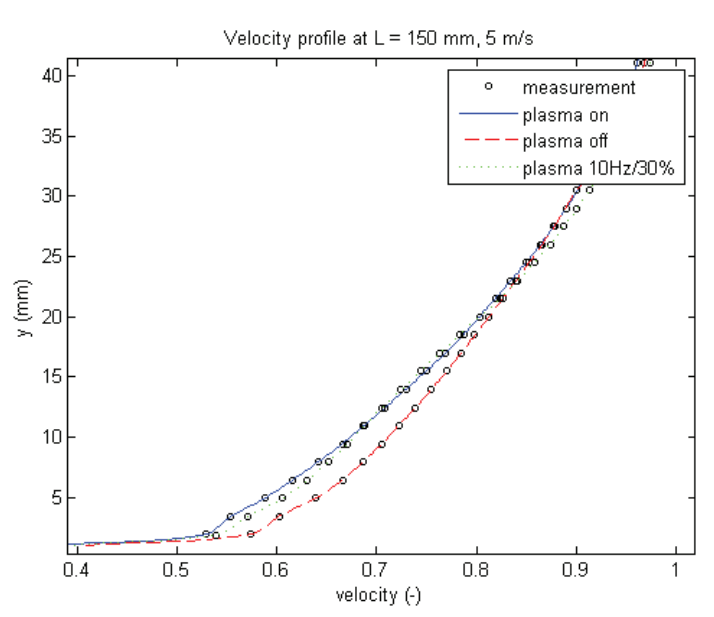

Figure 5. Effect of opposite direction of plasma on velocity profiles, long channel

There are no significant effects of actuation for plasma actuation in corresponding direction inside short channel. Since the thickness of boundary layer is very thin, the TR-PIV is not very convenient method. The space resolution of PIV is not satisfactory and the measurement is fraught with significant error in tight distance from the wall. This has to be taken into account.

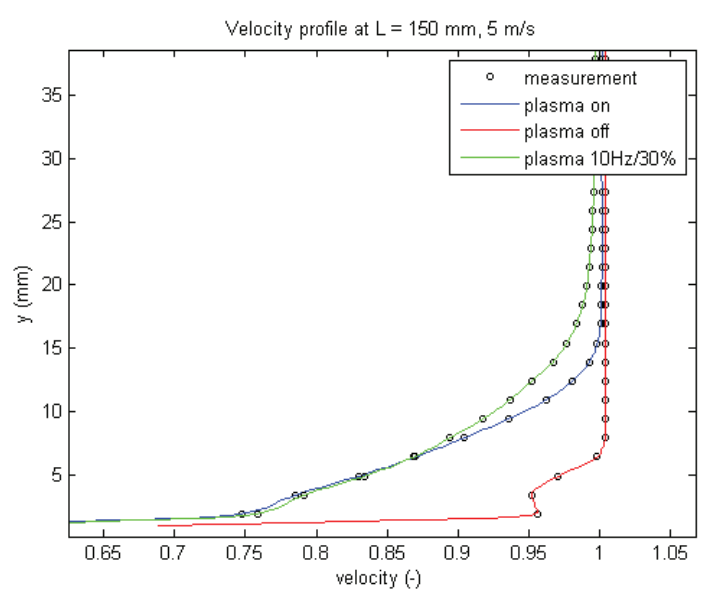

Figure 6. Effect of opposite direction of plasma on velocity profiles, short channel

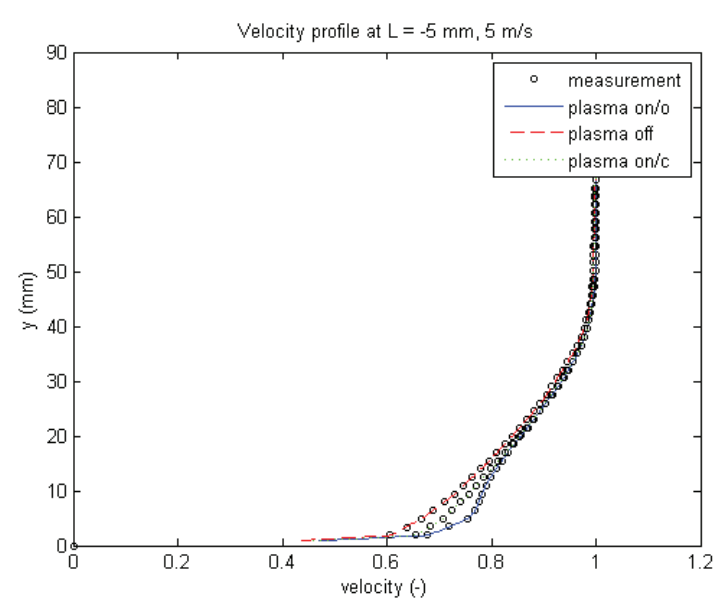

Figure 7. Effect of opposite direction of actuation upstream, long channel

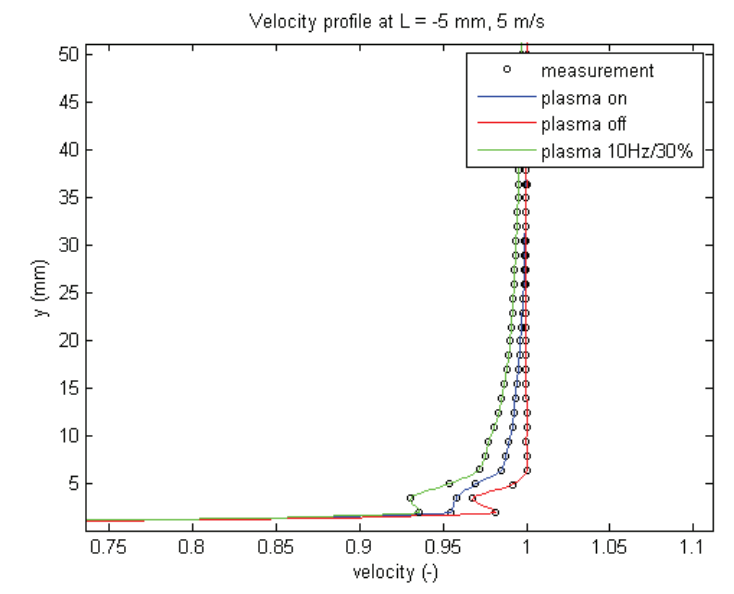

Figure 8. Effect of opposite direction of actuation upstream, short channel

The most interesting part is related with the influenced flow upstream the wire electrode $(\mathrm{L}=-5 \mathrm{~mm})$. The area under influence is very tiny. There is a slightly rise of velocities for plasma actuation oriented in corresponding direction (figure 7, green dashed line) and very strong increase of velocities for opposite orientation of actuator (figure 7, blue solid line). However, the situation is completely different for BL developed in shorter channel (figure 8). Here, all configuration of actuation resulted in significant degradation of velocity profile. This area is very complicated to measure due to existence of strong light effects. More, since there is a very strong electrostatic field between both electrodes, the seeding particles could be strongly deviated from the nature course of streamlines and acquired data do not agree with reality. This aspect will be subjugated under investigation in detail.

\subsection{Dynamical approach}

The data acquisition was time resolved (acquisition frequency $2000 \mathrm{~Hz}$ ), hence the dynamical aspect of interaction between boundary layer and induced flow was studied using Oscillation Pattern Decomposition (OPD) method. This method is based on stability analysis of the mean flow. One OPD mode is comprised from a eigenmode and a eigenvalue, both are very often represented by a complex number. The complex eigenmode represents a structure or pattern moving in space in a cyclic manner. The structure can be typically a wave or travelling vortex propagating in space. Complex eigenvalue contains information on the cyclical phenomenon frequency and decay in time [6]. The content of the frequency is one of the biggest advantage of this decomposition method.

The importance of each mode is evaluated by two quantities. One is e-folding time which can represent formally the average time for an amplitude of strength 1 to reduce to $1 / \mathrm{e}$. The periodicity is rate between e-folding time and oscillating period. The threshold of periodicity is 0.434 . The mode, whose value of periodicity is bigger than threshold, are true-oscillating modes. The rest of the modes are non-oscillating. Travelling modes (waves) are 
present in the flow that is characterized by convective velocity. On the other hand, pulsating modes are not moving in space.

The investigated position downstream was chosen so, that vortical structures generated by actuator were not fully merged with BL (position 1 of camera). The figure 9 shows the frequency spectrum of the most important modes in dependency on e-folding time for four regimes (blue - unsteady by $10 \mathrm{~Hz}$, red - unsteady by $20 \mathrm{~Hz}$, violet - steady regime, green - base case). The plotted modes (figures 10-13) are the most important modes for each individual configuration and were chosen so that their frequency is as close as possible to the frequency of modulation $-10 \mathrm{~Hz}$.

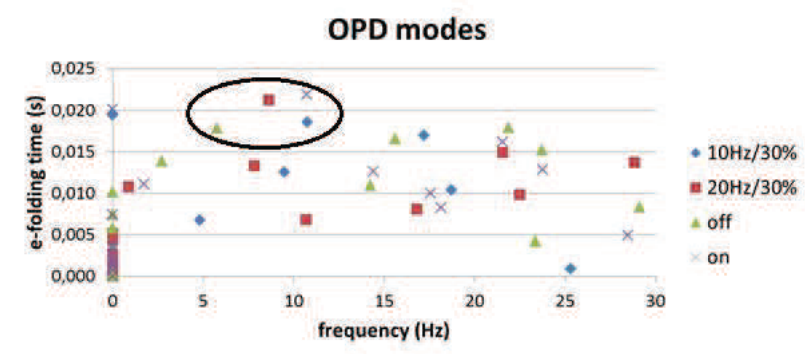

Figure 9. OPD modes up to $30 \mathrm{~Hz}$ in dependency on e-folding time for 4 configurations - plasma off, on and under 10 and 20 $\mathrm{Hz}$

All modes are in the form of propagating waves which is evident because main feature of that investigated case is convective motion. Modes are interpreted using streamlines to make visible all structures. Notice, that the direction of streamlines is not important because the analysis is coming out from fluctuation velocity components. Just a real part of modes is plotted (imaginary part of mode used to be shifted in space). The representation of modes as an animation is much more suitable because then the mode can be projected in complex space. Nevertheless, it can be seen at the figure 12 that mode contains a visible vortex which should be incorporated into the flow field by the modulation frequency of $10 \mathrm{~Hz}$.

The mode frequency of that unsteady case is $10.7 \mathrm{~Hz}$ which is almost the same value as the modulation frequency. The mode frequency of steady case (plasma on) exhibits the same value. The base case generated mode with frequency of $5.7 \mathrm{~Hz}$ and modulation by $20 \mathrm{~Hz}$ generated mode with frequency of $8.6 \mathrm{~Hz}$. This decomposition method is very sensitive to the accuracy of acquired and evaluated data and it should be treated with gained results rather carefully.

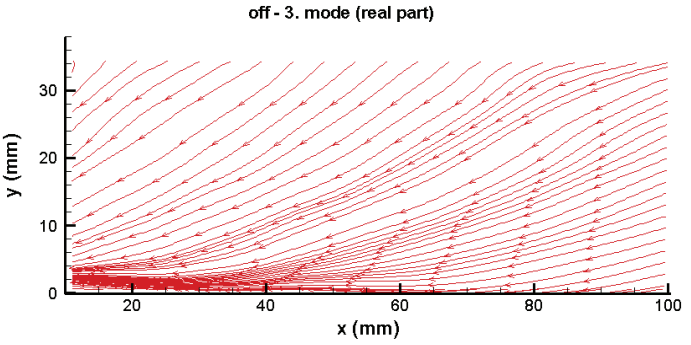

Figure 10. Real part of third mode, plasma actuation off, first position

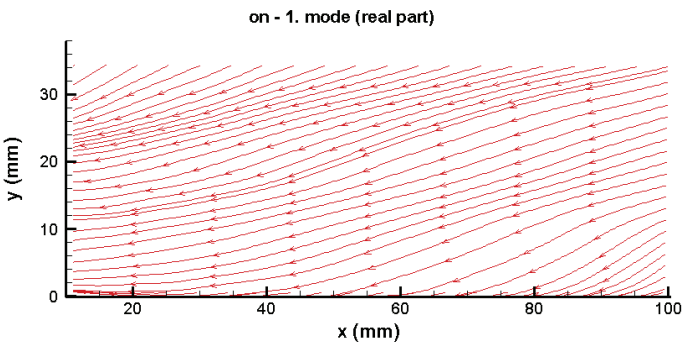

Figure 11. Real part of first mode, plasma actuation on, first position

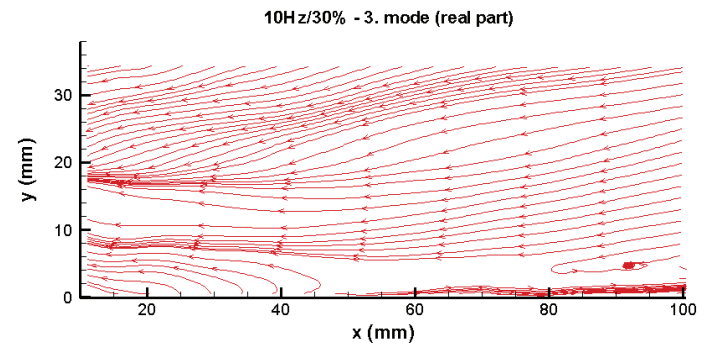

Figure 12. Real part of third mode, plasma generated by $10 \mathrm{~Hz}$, first position

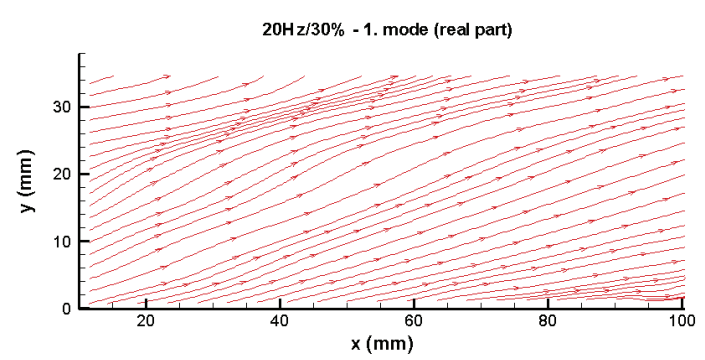

Figure 13. Real part of first mode, plasma generated by $20 \mathrm{~Hz}$, first position

\section{Conclusions}

Generated flow field by plasma dielectric barrier discharge actuator was measured by time resolved PIV and acquired data were threated in two different ways. From statistical point of view, it was found out that plasma ionic wind can improve significantly velocity profiles if the ionic wind is directed in corresponding 
orientation. Also, the velocity standard deviations became smaller which implies that plasma actuation can stabilize the flow inside the boundary layer. There are some unanswered questions and unclear phenomena in the vicinity of wire electrode. The particles are probably affected by strong electrostatic field.

Some preliminary results from OPD analysis were shown. The interpretation of complex modes is not easy task and the research in the field of dynamics will continue. One coherent structure was detected for unsteady case (modulation by $10 \mathrm{~Hz}$ ) and it is obvious that this vortex should be introduced by modulation frequency.

\section{Acknowledgement}

The authors gratefully acknowledge financial support of the Grant Agency of the Czech Republic, No. GP14$25354 \mathrm{P}$.

\section{References}

1. T. Abe, Y. Takizawa, S. Sato, AIAA Journal 46, 2248-2256 (2008)

2. P. Procházka, Vortex structures generated by plasma DBD actuator, Dissertation thesis, ČVUT (2013)

3. P. Procházka, V. Uruba, Exp. Fluid Mech., 594-598 (2013)

4. P. Procházka, V. Uruba, Proc. in Appl. Mathematics and Mechanics, 1, 667-668 (2011)

5. P. Jonáš, O. Mazur, V. Uruba, IV. Watermanagement conference, Praha (2004)

6. Dantec Dynamics, DynamicStudio manual (2012) 